\title{
Cyclin B1 is localized to unattached kinetochores and contributes to efficient microtubule attachment and proper chromosome alignment during mitosis
}

\author{
Qiang Chen ${ }^{1}$, Xiaoyan Zhang ${ }^{1}$, Qing Jiang ${ }^{1}$, Paul R Clarke ${ }^{2}$, Chuanmao Zhang ${ }^{1}$ \\ ${ }^{1}$ The MOE Key Laboratory of Cell Proliferation and Differentiation and the State Key Laboratory of Bio-membrane and Membrane \\ Bio-engineering, College of Life Sciences, Peking University, Beijing 100871, China, ${ }^{2}$ Biomedical Research Centre, College of Medicine, \\ Dentistry and Nursing, Level 5, Ninewells Hospital and Medical School, University of Dundee, Dundee DD1 9SY, Scotland, UK
}

Cyclin B1 is a key regulatory protein controlling cell cycle progression in vertebrates. Cyclin B1 binds CDK1, a cyclin-dependent kinase catalytic subunit, forming a complex that orchestrates mitosis through phosphorylation of key proteins. Cyclin B1 regulates both the activation of CDK1 and its subcellular localization, which may be critical for substrate selection. Here, we demonstrate that cyclin B1 is concentrated on the outer plate of the kinetochore during prometaphase. This localization requires the cyclin box region of the protein. Cyclin $\mathrm{B} 1$ is displaced from individual kinetochores to the spindle poles by microtubule attachment to the kinetochores, and this displacement is dependent on the dynein/dynactin complex. Depletion of cyclin B1 by vector-based siRNA causes inefficient attachment between kinetochores and microtubules, and chromosome alignment defects, and delays the onset of anaphase. We conclude that cyclin B1 accumulates at kinetochores during prometaphase, where it contributes to the correct attachment of microtubules to kinetochores and efficient alignment of the chromosomes, most likely through localized phosphorylation of specific substrates by cyclin B1-CDK1. Cyclin B1 is then transported from each kinetochore as microtubule attachment is completed, and this relocalization may redirect the activity of cyclin B1-CDK1 and contribute to inactivation of the spindle assembly checkpoint.

Keywords: cyclin B1, kinetochore, dynein, chromosome alignment, microtubule attachment

Cell Research (2008) 18:268-280. doi: 10.1038/cr.2008.11; published online 15 January 2008

\section{Introduction}

Progression through the cell cycle is driven by cyclindependent kinases (CDKs) which phosphorylate key substrates at specific stages of the cell cycle [1,2]. B-type cyclins form transiently active kinase complexes with CDK1 that have distinct roles in mitosis [1]. Cyclin B1 accummulates during S-G2 and shuttles between the cytoplasm and nucleus, becoming concentrated in the nucleus in late $\mathrm{G} 2$, and is then localized to centrosomes, mitotic spindle and chromosomes during prometaphase [3-7]. Cyclin B1 is degraded after completion of microtubule attachment to kinetochores during mitosis and inactivation of the spindle assembly checkpoint. In contrast to cyclin B1, cyclin B2 localizes to the Golgi apparatus in interphase, and is dis-

Correspondence: Chuanmao Zhang

E-mail: zhangcm@pku.edu.cn

Received 29 November 2007; revised 11 December 2007; accepted 16 December 2007; published online 15 January 2008 persed in mitosis with a small fraction on the spindle $[7$, 8]. The specific functions of B-type cyclins are likely to be determined by the specific localization of their complexes with CDK1 [1, 2, 7], resulting in the phosphorylation of multiple proteins controlling nuclear envelope breakdown (NEBD), mitotic spindle assembly, chromosome condensation and organelle reorganization during mitosis $[2,9]$.

Mammalian kinetochores are large protein complexes that are formed on chromosome regions known as centromeres. Kinetochores have a trilaminar structure, comprising an inner kinetochore plate directly adjacent to the centromeric heterochromatin and an outer plate with the associated corona [10]. The major function of the outer kinetochore domain involves the attachment of kinetochore fibres (k-fibres) formed by microtubules during mitosis. The formation of kinetochore-microtubule attachments during prometaphase is dynamic and involves a number of proteins [11-14], including a complex comprised of Hec1/Ndc80, Nuf2, Spc24 and Spc25 [14-16], several Ranregulated proteins (RanGAP1, RanBP2, HURP and Cenp 
F) [17-20] and microtubule plus-end tracking proteins, such as Ch-Tog, Clip-170, EB1 and the adenomatous polyposis coli (APC) $[13,21,22]$.

Kinetochores also generate a signal that prevents premature anaphase onset until all the chromosomes are correctly aligned on the spindle. This signal is part of the spindle checkpoint mechanism, a highly conserved mitotic checkpoint that ensures accurate chromosome segregation. The spindle checkpoint is silenced after all kinetochores have undergone bipolar attachment and thus contributes to the error-free segregation of chromosomes. The outer kinetochore domain also comprises several proteins that function in the spindle assembly checkpoint. The spindle checkpoint is mediated by a set of highly conserved kinetochore proteins including Mad1, Mad2, Mad3/BubR1, Bub1, Bub3 and Mps1 [11]. Unattached kinetochores accumulate Mad and Bub proteins and the downstream target of these checkpoint proteins is $\mathrm{Cdc} 20$, which is an activator of the anaphase-promoting complex/cyclosome $(\mathrm{APC} / \mathrm{C})$, an E3 ubiquitin ligase. Mad2 and BubR1 bind to $\mathrm{Cdc} 20$ and prevent it from activating $\mathrm{APC} / \mathrm{C}$, hence inhibiting the ubiquitination and degradation of cyclin B1 and securin, thereby inhibiting chromosome segregation and progression into anaphase.

Although kinetochore attachment of microtubules and mitotic spindle assembly are induced by cyclin B1-CDK1, the underlying mechanism is not yet fully understood. In part, the process involves indirect phosphorylation of substrates through activation of secondary kinases such as Aurora B and Plk1 [23], but also direct phosphorylation by cyclin B1-CDK1 of kinetochore-associated proteins, such as Cdc20 [23]. When phosphorylated by cyclin B1CDK1, Cdc20 interacts with Mad2 rather than APC/C and hence regulates the spindle checkpoint. Cyclin B1-CDK1 also regulates the kinetochore localization of Plk1 through phosphorylation of the kinetochore proteins Bub1 and Incenp [24, 25]. Plk1 modulates the association of spindle checkpoint proteins at kinetochores [26] and facilitates chromosome alignment during mitosis through phosphorylation of BubR1 [27, 28]. Further potential cyclin B1-CDK1 substrates localized at kinetochores are APC/C subunits [1, 2, 29] and RanGAP1 [30]. Interestingly, CDK1 had been shown to localize to kinetochores during mitosis [31], but the relevant cyclin partner awaited identification.

In this study, we show that the cyclin B1 is localized on the outer plate of kinetochores during prometaphase. Cyclin B1 is released from the kinetochores after microtubule attachment through dynein/dynactin-mediated transport along microtubules, with the same dynamics as spindle checkpoint proteins. Depletion of cyclin B1 disrupts the functions of the spindle checkpoint and the alignment of chromosomes during mitosis. Our study identifies a potential novel role of kinetochore-localized cyclin B1 in mitosis.

\section{Results}

Cyclin B1 localizes on the outer plate of kinetochores during mitosis

Cyclin B1 localizes to spindles [8] and chromosomes [3] during mitosis in several vertebrate species. We observed that cyclin B1 also localizes to kinetochores during mitosis using several approaches. Firstly, we immunostained HeLa cells with a mouse anti-cyclin B1 antibody (Supplementary information, figure S1A) and observed the kinetochore localization of cyclin B1 (Figure 1A). To confirm this kinetochore localization, we expressed a GFP-cyclin B1 fusion protein in HeLa cells and found that it co-localized with Cenp A, a kinetochore marker protein (Figure 1B). In live cell imaging experiments, we observed that GFP-cyclin B1 was strongly concentrated at kinetochores immediately after NEBD (Figure 1C). We also followed the process of kinetochore localization of cyclin B1 from prophase to metaphase. As shown in Figure 1A, cyclin B1 dramatically translocated into the nucleus at prophase as expected and a fraction of this protein began to localize to kinetochores. At prometaphase, the kinetochore localization of cyclin B1 became clearer. At metaphase, cyclin B1 disassociated from kinetochores, in contrast to its distinct localization to the mitotic spindle (Figure 1A).

Using co-immunostaining with an anti-Plk1 antibody, we observed that both cyclin B1 and Plk1 shared similar behaviours of localization to kinetochores (Supplementary information, figure S2). By expressing the GFP-cyclin B1, we also observed the dynamic localization of the exogenous fusion protein to kinetochores (Supplementary information, figure S3). To examine the relationship between the localization of cyclin B1 to chromosomes and its concentration at kinetochores, we performed chromosomal spread experiments and observed that cyclin B1 began to appear at kinetochores in late prophase or early prometaphase as well as at chromosomes with tightly adherent chromatid arms. The level of cyclin B1 at kinetochores peaked in prometaphase and disappeared at metaphase when chromatid arms were separated, but cyclin B1 at chromosome arms remained (Figure 1D). We observed similar results when using HeLa cells expressing GFP-cyclin B1 (Supplementary information, figure S4).

As mammalian kinetochores have a trilaminar structure, we sought to determine which part of the kinetochores cyclin B1 localized to. Plk1 has been reported to localize on the outer plate of kinetochores during mitosis [26]. We co-immunostained the cells with cyclin B1 and Plk1 antibodies and found that both proteins co-localized on 


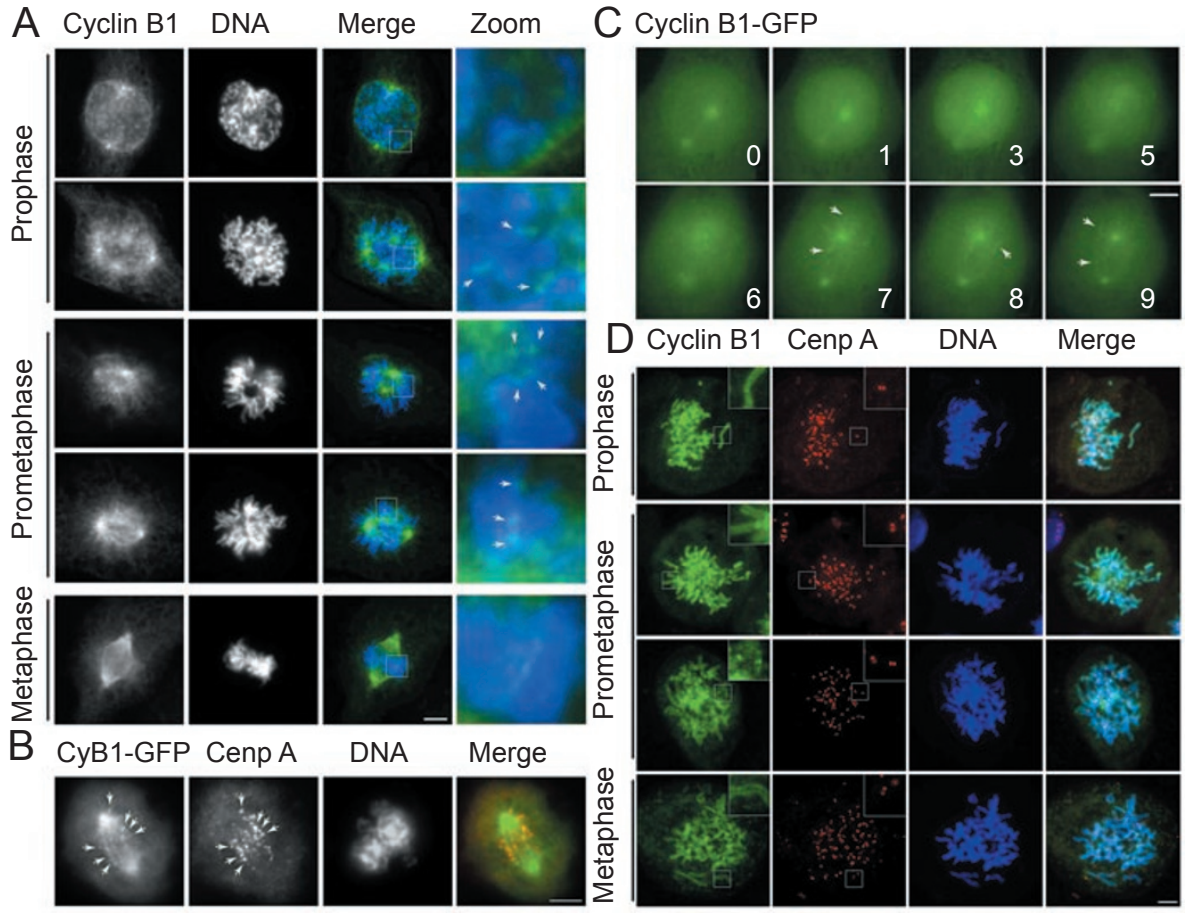

Figure 1 Kinetochore localization of cyclin B1. (A) HeLa cells were immunostained by anti-cyclin B1 antibody. The selected regions were magnified and the kinetochore localization of cyclin B1 was judged by its immunostaining patterns with paired dots on both sides of each chromosome as indicated by arrows. Bar $=5 \mu \mathrm{m}$. (B) HeLa cells expressing GFP-cyclin B1 were fixed with methanol and stained with anti-Cenp A antibody to indicate kinetochores. The kinetochore localization of GFP-cyclin B1 is indicated by arrows. Bar $=5 \mu \mathrm{m}$. (C) Still images of time lapse experiments with HeLa cells expressing GFP-cyclin B1showing the kinetochore localization of cyclin B1 in prometaphase. The kinetochore localization of cyclin B1 is indicated with arrows. Time is given in minutes. Bar $=5 \mu \mathrm{m}$. (D) Chromosomal spreads showing the localization of cyclin B1 on chromosomes and kinetochores from prophase to metaphase. Cenp A (red) was co-immunostained with cyclin B1 (green). Selected regions are magnified, showing the chromosome and kinetochore localization of cyclin B1. Bar $=5 \mu \mathrm{m}$.

the outer plate of kinetochores (Figure 2C). It has been reported that Aurora $\mathrm{B}$ localizes on the centromere region and HEC1 localizes on the outer plate of kinetochores [16]. By co-immunostaining GFP-cyclin B1 expressing HeLa cells with antibodies against Aurora B or HEC1, we found that GFP-cyclin B1 co-localized with HEC1 but not Aurora B (Figure 2A and 2B). These results indicated that a fraction of cyclin B1 molecules localize on the outer plate of kinetochores during mitosis. Cyclin B1 begins to accumulate on kinetochores in prophase and peaks in prometaphase. Once the cell enters metaphase, this fraction of cyclin B1 disappears from kinetochores.

Cyclin B1 disassociates from kinetochores after the attachment of microtubules to the kinetochores

To determine the relationship between the disappearance of cyclin B1 from kinetochores and the attachment of microtubules to the kinetochore in metaphase, we treated the cells with nocodazole to disassemble microtubules and observed that the kinetochore-localized cyclin B1 remained
(Figure 3A). Furthermore, in contrast to DMSO-treated control cells, nocodazole treatment caused an enhanced accumulation of cyclin B1 on kinetochores (Figure 3A). To re-establish the attachment of microtubule to kinetochores, nocodazole-treated cells were replated into fresh medium for $30 \mathrm{~min}$ and then fixed. Under these conditions, most chromosomes became aligned at the middle of the spindle with the re-establishment of attachment of microtubules to their kinetochores, while some chromosomes were left behind due to delayed attachment of microtubules. In these cells, we observed that cyclin B1 disappeared from kinetochores of aligned chromosomes, but remained on the kinetochores of unaligned chromosomes (Figure 3B). These results suggested that microtubule attachment to a kinetochore caused the disappearance of cyclin B1 specifically from that kinetochore during metaphase. This result might be explained by two mechanisms: one being that cyclin B1 is degraded specifically on microtubule-attached kinetochores, the other that cyclin B1 is transported away from the kinetochore. To distinguish between these pos- 


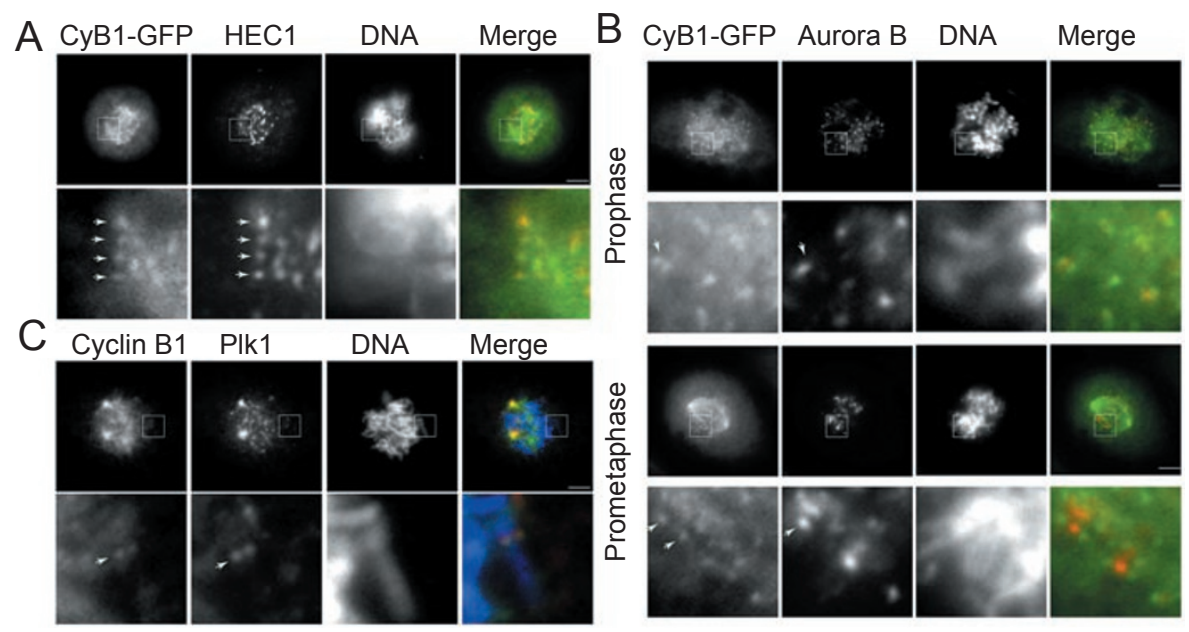

Figure 2 Cyclin B1 is localized on the outer plate of kinetochores. (A) Cyclin B1 co-localized with HEC1 on the outer plate of kinetochores. HeLa cells expressing GFP-cyclin B1 were fixed by methanol and stained with anti-HEC1 antibody. Selected regions are magnified showing the colocalization of cyclin B1 (originally green) and Hec1 (originally red). The kinetochore localization of cyclin B1 and HEC1 is indicated with arrows. Bar $=5 \mu \mathrm{m}$. (B) The localization of cyclin B1 compared with Aurora B on kinetochores. HeLa cells expressing GFP-cyclin B1 (originally green) were fixed and stained with anti-Aurora B antibody (originally red). Selected regions are magnified comparing the localization between cyclin B1 and Aurora B. The kinetochore localization of cyclin B1 and the centromere localization of Aurora B are indicated with arrows. (C) HeLa cells co-immunostained by anti-cyclin B1 (originally green) and anti-Plk1 (originally red) antibodies showing their colocalization. Bar $=5 \mu \mathrm{m}$.

sibilities, nocodazole-treated cells were replated in fresh medium lacking nocodazole for $30 \mathrm{~min}$. Cyclin B1 was diminished on the kinetochores of aligned chromosomes regardless of whether the proteosome inhibitor MG132 was present or not (Figure 3B), indicating that the disappearance of cyclin B1 from attached kinetochores was not due to proteosomal degradation; rather, cyclin B1 was likely transported away from a kinetochore after the establishment of microtubule attachment.

It has been shown that the outer plate kinetochore proteins Mad2 and BubR1 are transported along microtubules to spindle poles by the dynein/dynactin complex after the attachment of microtubules to the kinetochores [32], while HEC1 is not transported [16]. We therefore examined whether the dynein/dynactin complex is involved in the relocalization of cyclin B1 away from attached kinetochores. By immunostaining, we observed that cyclin B1 co-localized with dynactin to kinetochores and spindles during early mitosis (Supplementary information, figure S5). When ATP was depleted with sodium azide and deoxyglucose, a treatment that promotes dynein/dynactindependent transport of Mad2 and BubR1 from kinetochores [32-34], GFP-cyclin B1, but not HEC1, left the kinetochores and concentrated on the spindle poles (Figure 3C). By contrast, when cells were pretreated with nocodazole to disassemble the microtubules, and then exposed to sodium azide and deoxyglucose, GFP-cyclin B1 did not leave the kinetochores and still co-localized with HEC1 (Figure 3C). These results suggest that cyclin B1 is transported from kinetochores to the spindle poles and its transport involves the dynein/dynactin complex.

\section{The cyclin box domain is required for the kinetochore} localization of cyclin B1

To determine which domain of cyclin B1 is responsible for the localization on kinetochores, we constructed a number of GFP-tagged cyclin B1 truncations and point mutants (Figure 4A) and introduced them into HeLa cells. We observed that $\mathrm{N}$-terminal domains consisting of amino acids 1-90 or 1-200 that lacked the entire cyclin box, which is required for interaction with $\mathrm{CDK} 1[35,36]$, could not localize to kinetochores, while a truncation consisting of amino acids 150-433 containing the cyclin box localized to kinetochores like full-length cyclin B1. These results suggested that the cyclin box is important for the localization of cyclin B1 on kinetochores. To test this notion further, we generated an R202A mutant and a $\mathrm{C} \Delta 24$ truncation to disrupt the function of the cyclin box $[35,36]$ and introduced them into HeLa cells. In these mutants, the kinetochore localization of cyclin B1 was completely disrupted (Figure 4B). Therefore, the cyclin box of cyclin B1 is required for its localization to kinetochores.

We also noticed that the ability of the N-terminal deletion mutant consisting of amino acids $150-433$ to localize 

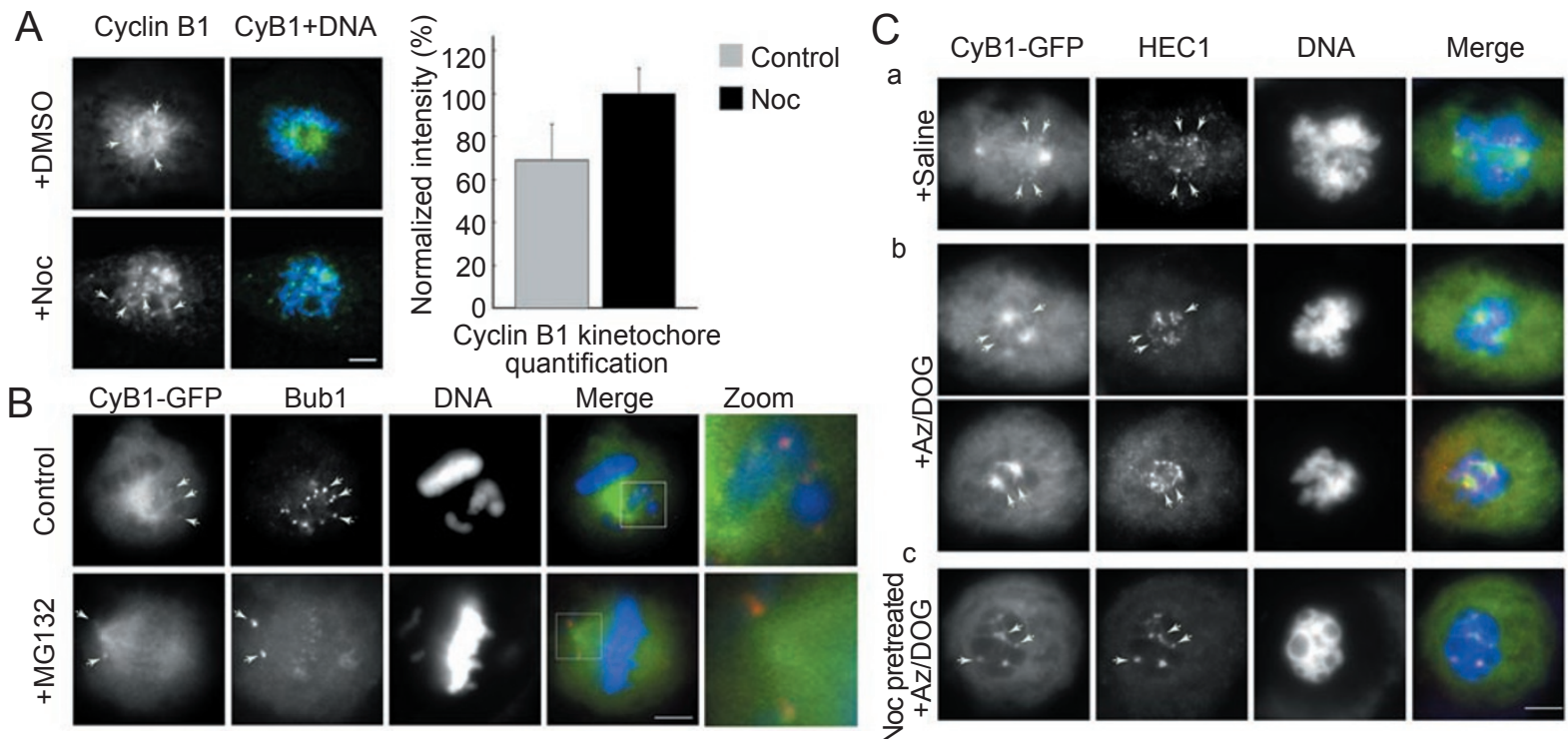

Figure 3 Displacement of cyclin B1 from kinetochores by microtubule attachment and the role of the dynein/dynactin complex. (A) When HeLa cells were treated with nocodazole to disassemble microtubules, the accumulation of cyclin B1 at kinetochores was enhanced. Control (DMSO) and nocodazole-treated cells were fixed and immunostained with a mouse anti-cyclin B1 antibody. The kinetochore localization of cyclin B1 is determined by its immunostaining patterns with paired dots on both sides of each chromosome and is indicated with arrows. The mean intensity of cyclin B1 on kinetochores was shown in both DMSO- and nocodazole-treated cells ( $n>100$ pairs of kinetochores in 8 cells in each category). Error bars indicate SD. Bar $=$ $5 \mu \mathrm{m}$. (B) HeLa cells expressing GFP-cyclin B1 were blocked in prometaphase by nocodazole treatment for $2 \mathrm{~h}$ and released for $1 \mathrm{~h}$ under the treatment of DMSO (control) or MG132. Cells with several unaligned chromosomes were selected to show the localization of cyclin B1 on the unattached kinetochores in contrast to the microtubule-attached kinetochores. Bub1 was immunostained to show the unattached kinetochores (red). A selected region is magnified to show the kinetochore localization of cyclin B1. Unattached kinetochores are indicated with arrows. Bar $=5 \mu \mathrm{m}$. (C) The kinetochore localization of cyclin B1 was regulated by the dynein/dynactin complex. (a) Cells in a control experiment were treated with saline g (Saline). (b) Cells were depleted of ATP by treatment with azide and deoxyglucose (AZ/DOG). (c) Nocodazole pretreated cells were treated with azide and deoxyglucose (Noc pretreated + AZ/DOG). Cells were immunostained with anti-HEC1 antibody to show kinetochores (red). The kinetochore localization is indicated with an arrow. Bar $=5 \mu \mathrm{m}$.

to kinetochores was reduced compared to that of full-length cyclin B1 (Figure 4B), suggesting that the N-terminus might also contribute to cyclin B1 kinetochore localization. Through mitotic chromosomal spread experiments (Figure 4C), we observed, however, that the destructionbox (D-box) motif had no contribution to the cyclin B1 kinetochore localization, since $\mathrm{R} 42 \mathrm{~A}$ and $\Delta \mathrm{D}$-box mutants were indistinguishable from full-length wild type cyclin B1. Another possible mechanism for recruitment of cyclin B1 to the kinetochore might be through its nuclear export signal (NES) located in the N-terminal portion of the protein, since the NES-binding transport factor Crm1 localizes to kinetochores during mitosis and recruits certain NES-containing proteins. This interaction is disrupted by leptomycin B (LMB) [37]. We therefore treated cells with $\mathrm{LMB}$, but found no effect on the kinetochore localization of cyclin B1 in mitotic cells while cyclin B1 was concentrated in the nuclei of interphase cells due to inhibition of
Crm1-mediated nuclear export (Supplementary information, figure S6A). This result indicated that an interaction between $\mathrm{Crm} 1$ and the NES domain of cyclin B1 makes no contribution to kinetochore localization of cyclin B1. Deletion of residues 1-33 reduced the kinetochore localization of cyclin B1 and this effect was enhanced by deletion of residues 1-150 (Figure 4C); interestingly, these truncations similarly reduced the ability of cyclin B1 to associate with chromosomes (Supplementary information, figure S6C, E). The quantification of the ratio of the GFP fluorescence to the Cenp A signal for each mutant was shown in Supplementary information, figure S6D. We also checked the expression level of each mutant and found no major differences (Supplementary information, figure S7). Together these results indicate that the N-terminal portion of cyclin B1 could contribute to its kinetochore localization as well as its chromosome localization. 
A

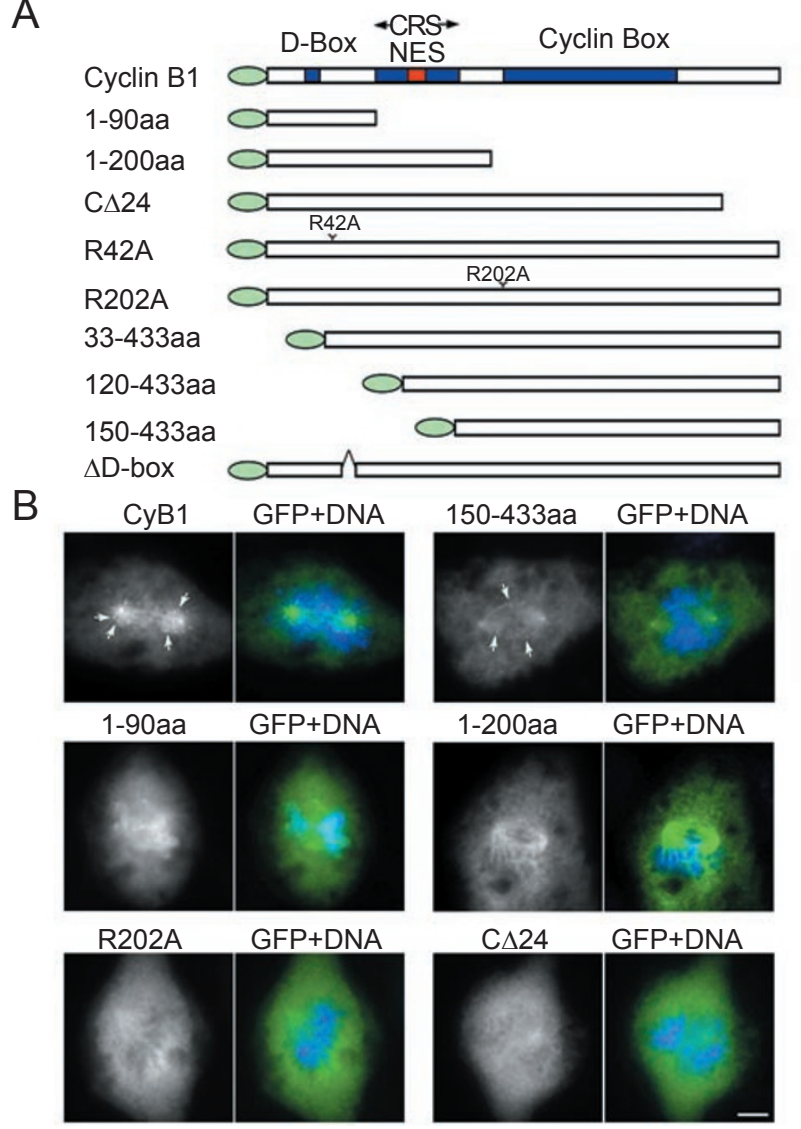

C
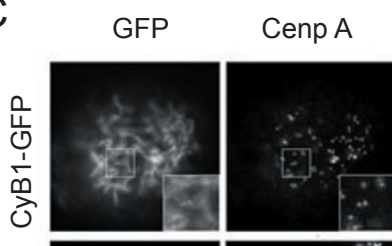

DNA

Merge
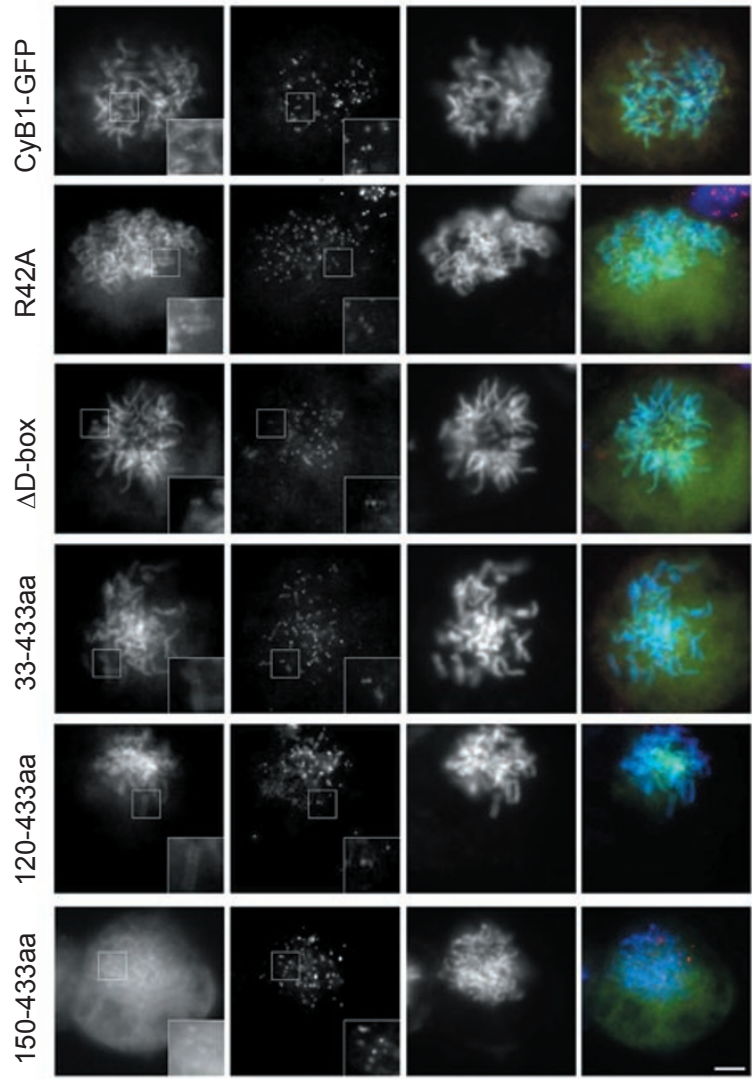

Figure 4 The cyclin box of cyclin B1 is required for the localization to kinetochores. (A) Diagram showing several mutants of cyclin B1, each fused with the GFP tag at the N-terminus. (B) A truncated mutant of cyclin B1 lacking the cyclin box could not localize on kinetochores. The kinetochore localization of cyclin B1 is determined by its immunostaining patterns with paired dots on both sides of each chromosome. Truncated mutants, 1-90aa and 1-200aa of cyclin B1, expressed as GFP fusions could not localize on kinetochores in contrast to full-length cyclin B1. The accumulation of one truncation (150-433aa) on kinetochores was weaker than that of full-length cyclin B1. The kinetochore localization of cyclin B1 proteins is indicated with arrows. Bar = $5 \mu \mathrm{m}$. (C) Kinetochore localization of cyclin B1 mutants harboring deletions or point mutation at the N-terminus (green) on a chromosome spread. Cells were immunostained with anti-CenpA antibody to indicate kinetochores (red). Selected regions were magnified, showing the kinetochore localization of cyclin B1 mutants. Bar $=5 \mu \mathrm{m}$.

Depletion of cyclin B1 delays chromosome alignment during mitosis

To test the possible function of cyclin B1/CDK1 at kinetochores, we depleted the endogenous cyclin B1 of HeLa cells using pSUPER vector-based siRNA (pS-cyclin B1). At $48 \mathrm{~h}$ after $\mathrm{pS}$-cyclin B1 transfection, the cyclin B1 protein level was decreased substantially as detected by immunoblotting (Figure 5A), whereas cyclins A2 and B2 were not affected (Supplementary information, figure S8). For immunostaining, we co-transfected HeLa cells with pS-cyclin B1 and GFP-H2B (serving as a transfection-positive marker) at a ratio of 20:1. At $48 \mathrm{~h}$ after transfection, we found no detectable cyclin B1 in GFP-H2B-positive cells (Figure 5A). Strikingly, we observed that, in contrast to control cells, of which about 5\% exhibited misaligned chromosomes, about $25 \%$ of cyclin B1-depleted metaphase cells contained at least one unaligned chromosome. The unaligned chromosomes were often located near spindle poles, suggesting that the attachment of microtubules to kinetochores was not properly established on these unaligned chromosomes (Figure 5B). We immunostained the spindle checkpoint protein Bub1 and found that kinetochores of unaligned chromosomes in cyclin B1-depleted cells had clearly higher Bub1 levels than those of aligned chromosomes (Figure 5C). To confirm if the kinetochore attachment of microtubules was affected by cyclin B1 depletion, we treated cells with ice-cold conditions for $10 \mathrm{~min}$ and found that the kinetochore microtubules (k-fibres) were less 


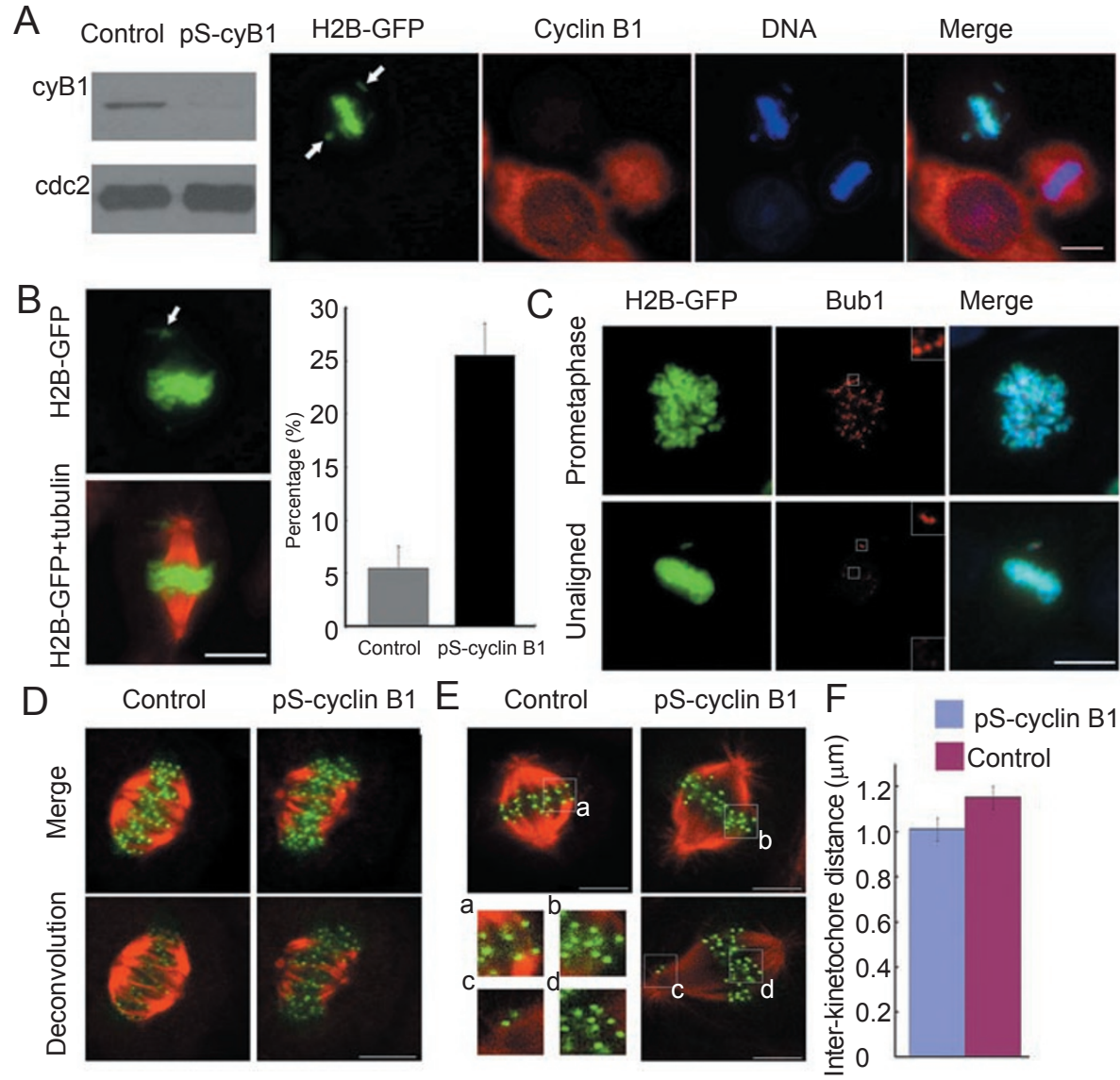

Figure 5 Cyclin B1 is required for the stable attachment of microtubules to kinetochores. (A) HeLa cells transfected with control or pSuper-cyclin B1 (cyclin B1 RNAi) were collected $48 \mathrm{~h}$ after transfection and analyzed by western blotting with cdc2 serving as the loading control (left). GFP-H2B and pSuper-cyclin B1 co-transfected (1:20) metaphase cells were immunostained with anti-cyclin B1 antibody (red); and the unaligned chromosomes are indicated with arrows (right). Bar $=10 \mu \mathrm{m}$. (B) The percentage of metaphase cells with unaligned chromosomes was quantified in control $(n>500)$ or cyclin B1-depleted $(n>500)$ cells. Each experiment was repeated three times. Error bars represent SD. Arrow indicates unaligned chromosomes. Bar $=10 \mu \mathrm{m}$. (C) Images of cyclin B1-depleted HeLa cells stained for Bub1 (red). GFP-H2B was co-transfected with pSuper-cyclin B1 at a ratio of 1:20. Prometaphase and metaphase with unaligned chromosomes are shown. Selected regions were magnified to show the intensity of Bub1 on prometaphase chromosomes, unaligned chromosome and aligned chromosomes. Bar $=10 \mu \mathrm{m}$. (D) Control cells (control) and cells with depletion of cyclin B1 (pS-cyclin B1) were treated with ice-cold conditions for 10 min and fixed to be immunostained with anti- $\alpha$-tubulin antibody (red) and observed by confocal microscopy. The merged image of z-stacks is shown in the upper panel and a sole deconvolution image of the same cell is shown in the lower panel. Bar $=10$ $\mu \mathrm{m}$. (E) Control and pS-cyclin B1 transfected cells were immunostained with anti- $\alpha$-tubulin antibody (red) and were observed by confocal microscopy. GFP-Cenp A was transfected to show paired kinetochores. Selected regions were magnified and are shown. Bar $=10 \mu \mathrm{m}$. (F) Interkinetochore distance was determined by the distance of paired GFP-Cenp A signals. Over 100 pairs of kinetochores were scaled in at least 20 cells (average of three independent experiments). Error bars represent SD.

stable in cyclin B1-depleted cells comparing with control cells (Figure 5D). We also measured the inter-kinetochore distances of paired kinetochores and found that the distance in cyclin B1-depleted cells $(1.03 \mu \mathrm{m})$ was significantly less than in control cells $(1.12 \mu \mathrm{m})$ (Figure $5 \mathrm{E}$ and $5 \mathrm{~F}$ ). These results indicate that cyclin B1 depletion affects the microtubule capture ability of kinetochores and imply that cyclin B1-CDK1 functions in the correct attachment of microtubules to kinetochores.

To determine if the chromosome alignment defects affected mitotic progression, we followed the mitotic process of cyclin B1-depleted cells by time-lapse microscopy. In a control cell, the time from NEBD to full metaphase chromosome alignment lasted about $20 \mathrm{~min}$, while in two cyclin B1-depleted cells, which exhibited lagging chromosomes, the process took about $60 \mathrm{~min}$ (Figure 6A). When 
the process was measured in many cells with or without lagging chromosomes, the mean time from NEBD to full chromosome alignment was $32 \mathrm{~min}$ in cyclin B1-depleted $(\mathrm{n}=78)$ cells and $20 \mathrm{~min}$ in control cells $(\mathrm{n}=50)$ (Figure 6B). Remarkably, only $51 \%$ of cyclin B1-depleted cells observed could complete chromosome alignment within 30 min, while $84 \%$ of control cells finished their chromosome alignment within this time. Thus, $49 \%$ of cyclin B1-depleted cells took more than $30 \mathrm{~min}$ for their chromosomes to align in contrast to $16 \%$ of control cells. More than $10 \%$ of cyclin B1-depleted cells took 60 min or more to finish chromosome alignment (Figure 6C). These results show that depletion of cyclin $\mathrm{B} 1$ affects chromosome alignment and mitotic progression, although the process of NEBD and spindle assembly still occurs.

\section{Cyclin B1-CDK1 regulates the function of the spindle checkpoint}

By live cell imaging experiments, we found that most HeLa cells depleted of cyclin B1 could enter anaphase eventually, though with delay of chromosome alignment, and, in some cases, cells with cyclin B1 depletion entered anaphase despite not all the chromosomes being aligned properly (Figure 7A). This indicated that, though the spindle checkpoint was activated due to the unaligned chromosomes, cells without cyclin B1 could not be restrained in

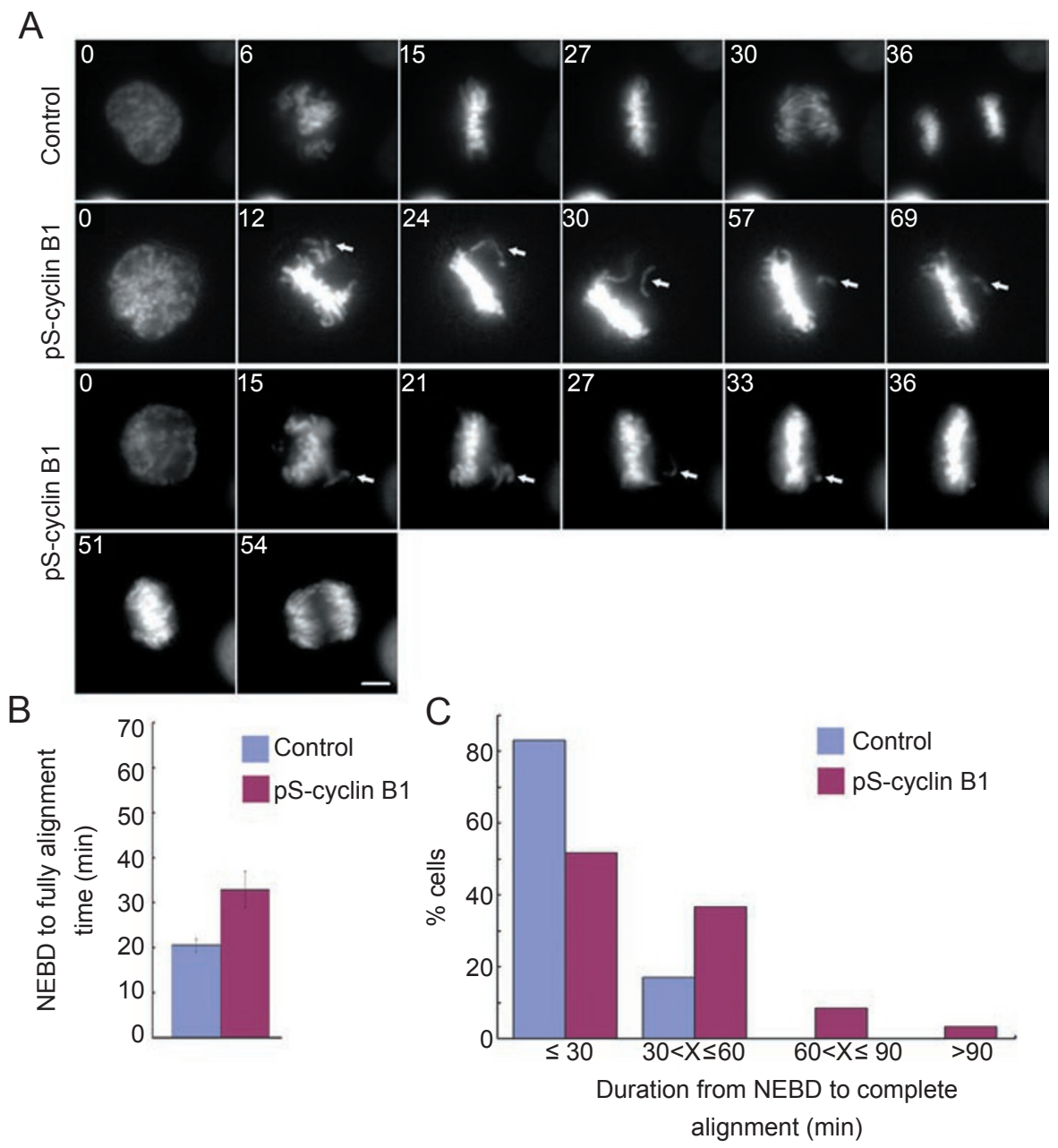

Figure 6 Depletion of cyclin B1 delays chromosome alignment. (A) Still frames from time-lapse microscopy of control (control) or cyclin B1-depleted (pS-cyclin B1) HeLa/GFP-H2B cells are listed. Time is given in minutes. Arrow indicates unaligned chromosomes. Bar $=5 \mu \mathrm{m}$. (B) The duration of different periods from prophase to anaphase onset was calculated from time-lapse movies of 50 control and 78 cyclin B1-depleted cells. HeLa/GFP-H2B cells were monitored by time-lapse microscopy using a very low level of light intensity to determine the duration of NEBD to metaphase with all chromosomes aligned to the equator (NEBD to alignment). Error bars represent SD. (C) Histograms showing the percentages of mitotic cells that had progressed within the indicated time frames from prophase to complete chromosome alignment. 

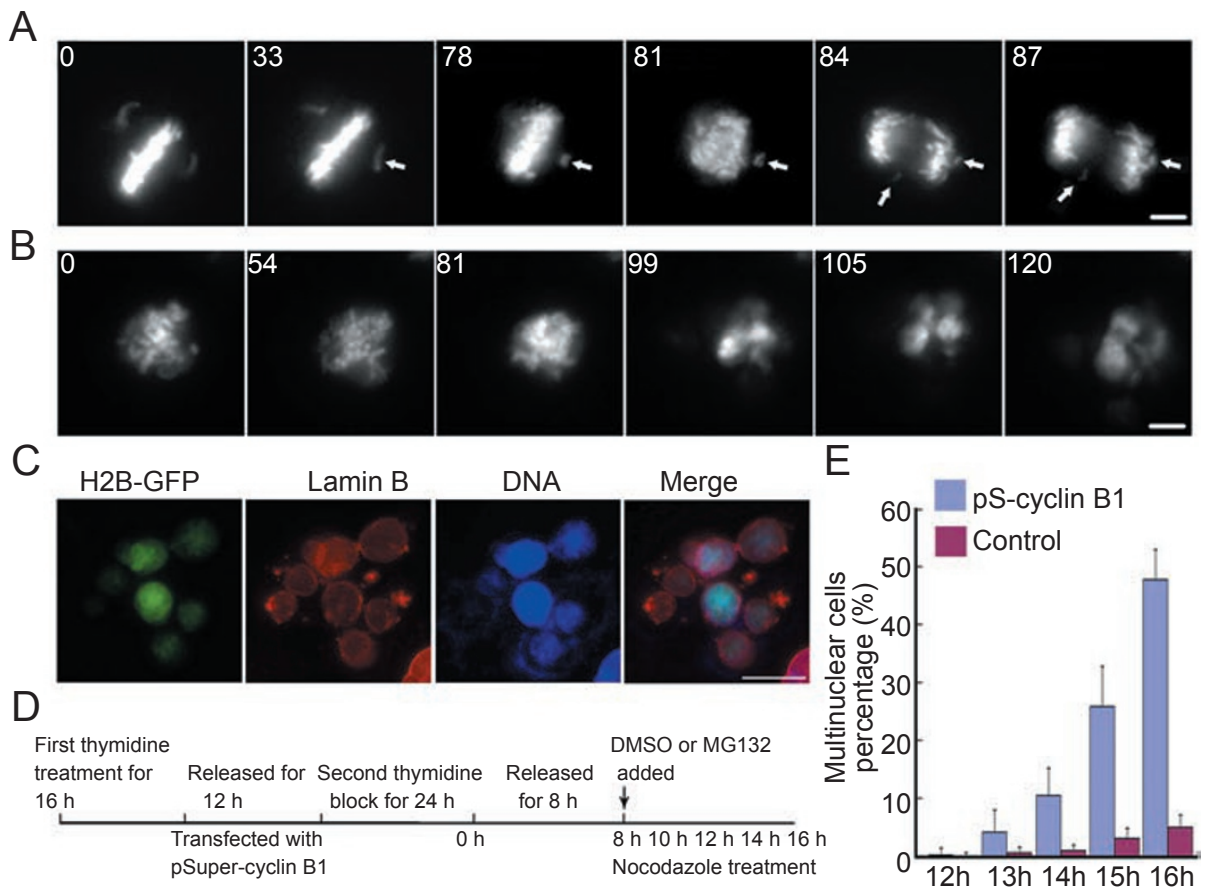

$\mathrm{F}$

Control

pS-cyclin B1
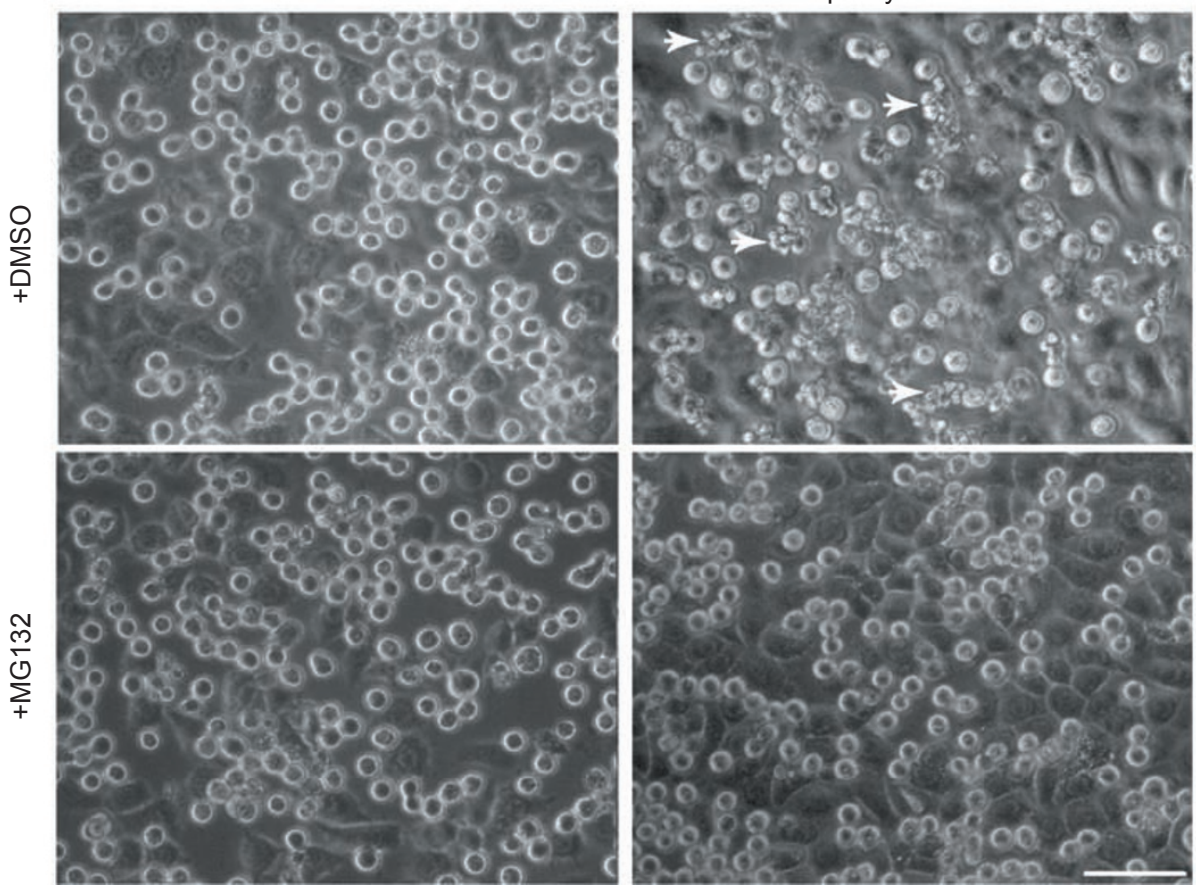

Figure 7 Depletion of cyclin B1 compromises the spindle checkpoint. (A) Still images of time-lapse microscopy of a HeLa/GFP$\mathrm{H} 2 \mathrm{~B}$ cell transfected with pSuper-cyclin B1. Anaphase onset was between 78-81 min. Arrows indicate unaligned chromosomes. Time is given in minutes. Bar $=5 \mu \mathrm{m}$. (B) Still images of a representative HeLa/GFP-H2B cell transfected with pSuper-cyclin $\mathrm{B} 1$ treated with $200 \mathrm{nM}$ nocodazole. Time is given in minutes. Bar $=5 \mu \mathrm{m}$. (C) A representative multinuclear cell caused by depletion of cyclin B1 was immunostained with anti-lamin B antibody to show the interphase nuclear membrane. Bar $=10 \mu \mathrm{m}$. (D) Diagram showing experimental protocols used in (E) and (F). (E) The effect of depletion of cyclin B1 on nocodazole-treated cells. Multinuclear cells were quantified in control (control, $n>500$ ) or cyclin B1-depleted ( $p S$-cyclin B1, $n>500$ ) cells at each time point as indicated (average of three independent experiments). The time point at which cells were released from double thymidine block is indicated as 0 hour. The percentage of multinuclear cells at each time point listed in the figure is shown. (F) Phase contrast images of control and cyclin B1 depleted cells that were released from double thymidine block for $15 \mathrm{~h}$. DMSO or MG132 was added at the $8 \mathrm{~h}$ time point with nocodazole $(200 \mathrm{nM})$. Multinuclear cells are indicated with arrows. Bar $=100 \mu \mathrm{m}$. 
metaphase for a prolonged period. This suggested that the spindle checkpoint was affected by depletion of cyclin B1, though not fully abrogated. To address the effect of cyclin B1 on the spindle checkpoint, we first treated cyclin B1-depleted cells with nocodazole to block cells in prometaphase, then analysed these cells by live cell imaging. We found that cells without cyclin B1 escaped the nocodazole-induced mitotic arrest and entered G1 phase without chromosome segregation after $2 \mathrm{~h}$, resulting in multinuclear cells (Figure 7B) as determined by the nuclear morphology. To confirm the effect of cyclin B1 depletion on the spindle checkpoint, cells were treated as outlined schematically in Figure 7D. We found that control cells released from a double-thymidine block responded to nocodazole by arresting with a rounded morphology, reflecting the activation of the spindle checkpoint. In contrast, cells depleted of cyclin B1 were unable to sustain such an arrest. Instead, some of these cells flattened out (Figure 7F) and reformed interphase nuclei, as confirmed by anti-lamin B immunostaining (Figure 7C). We counted the percentage of multinuclear cells at each time point: more than $50 \%$ of cells with depletion of cyclin B1 escaped the spindle assembly checkpoint block caused by nocodazole treatment using this protocol in contrast to less than $10 \%$ in control cells (Figure 7E). To test further if this effect was due to the impairment of the spindle checkpoint and not a simple lacking of cyclin B1, we treated released cells with MG132 and found that cells with depletion of cyclin B1 could not enter G1 phase when the proteosomal degradation of other proteins was inhibited (Figure 7F). This indicates that cyclin B1 depletion also permits the degradation of other mitotic proteins and therefore implies that cyclin B1 is required for the maintenance of the spindle checkpoint, which otherwise restrains "premature" proteosomal degradation.

\section{Discussion}

The function of cyclin-dependent kinases depends on both their activity and their subcellular localization. In this study, we have shown that a fraction of the mitotic cyclin, cyclin B1, localizes to unattached kinetochores during prometaphase. When we were preparing our manuscript, a paper reporting similar findings of kinetochore localization of cyclin B1 was published online [38]. However, the paper by Bentley et al. did not report on the possible functions of cyclin B1-CDK1 on kinetochores. They also did not clarify the function of the dynein/dynactin complex in regulating the dynamics of cyclin B1 on kinetochores. By depletion of cyclin B1 expression through vector-based siRNA, we show that cyclin B1 may play a role in the attachment of microtubules to kinetochores and in the progression of mitosis. We have also provided evidence that cyclin B1 plays a role in maintenance of the spindle checkpoint that controls the timing of anaphase. These results suggest that the activity of cyclin B1-CDK1 kinase molecules localized to kinetochores may contribute to the attachment of microtubules to kinetochores and chromosome alignment, presumably through phosphorylation of co-localized substrates. Importantly, we have shown that cyclin B1 is transported away from individual kinetochores to the spindle poles as microtubule attachments to kinetochores are established. This transport occurs prior to global cyclin B1 degradation when all kinetochore attachments are completed and the spindle checkpoint is satisfied. Thus, the function of cyclin B1-CDK1 at kinetochores is likely to be controlled during progression through mitosis via changes in its co-localization with substrates at kinetochores, with an increasing presence at unattached kinetochores during prometaphase and subsequent transport of cyclin B1 away to the poles of the spindle during the transition to metaphase.

Previously, it has been reported that the cyclin B1-CDK1 complex phosphorylates several kinetochore or centromere proteins. In our study, we found that cyclin B1 is localized on kinetochores during early mitosis. One likely substrate for cyclin B1-CDK1 at unattached kinetochores is Cdc20, which when phosphorylated is bound by Mad2 so that the activity of APC/C is inhibited [23]. With the completion of microtubule attachment and chromosome alignment, Mad2 is released from the kinetochores and $\mathrm{Cdc} 20$ associates with $\mathrm{APC} / \mathrm{C}$ to activate its function as a ubiquitin ligase. This leads to poly-ubiquitylation of cyclin B1, securin, and other proteins, and their destruction by the proteosome, triggering the onset of anaphase. This scenario is consistent with our results showing that cyclin B1 is required for proper spindle checkpoint function in human cells and the works of others indicating a function for CDK1 in the spindle checkpoint in Xenopus extracts [23, 39]. BubR1 is also a likely substrate for cyclin B1-CDK1 on kinetochores. Recently, several papers $[28,40]$ reported that phosphorylation of BubR1 by cyclin B1-CDK1 facilitates the binding between BubR1 and Plk1 and the phosphorylation of BubR1 by Plk1. Phosphorylation of BubR1 by Plk1 generates the 3F3/2 phosphoepitope. Phosphorylation of BubR1 by cyclin B1-Cdk1 and Plx1 greatly enhances the kinase activity of BubR1 in vitro [40]. Thus, cyclin B1Cdk1 controls checkpoint arrest through phosphorylation of BubR1. Our results that depletion of cyclin B1 affected the spindle assembly are also consistent with this notion. Additional potential substrates for cyclin B1-CDK1 at kinetochores include Incenp, Bub1, APC/C subunits and RanGAP1, which may play roles in the establishment of kinetochore fibre attachments or regulation of the spindle checkpoint $[1,2,37]$. Cyclin B1-CDK1 may also regulate local protein phosphorylation indirectly through effects on 
downstream protein kinases or phosphatases co-localized at kinetochores.

Transport of cyclin B1 away from attached kinetochores to the spindle poles, apparently driven like Mad2 and BubR1 [32] through a dynein/dynactin-dependent process, could effectively prevent its ability to phosphorylate substrates remaining at kinetochores and promote spindle checkpoint inactivation. If cyclin B1 is transported as an active complex with CDK1, then it could subsequently phosphorylate new substrates on the spindle, thereby switching its function upon microtubule attachment to each kinetochore. This provides a potential mechanism for the control of cyclin B1-CDK1 during mitosis prior to its global inactivation when the spindle checkpoint is satisfied and cyclin B1 is degraded.

Cyclin B1 contains an N-terminal domain comprised of a CRS motif and D-box, and a C-terminal cyclin box. When we disrupted the cyclin box function by mutations, the localization of mutant cyclin B1 on kinetochores disappeared. Since the cyclin box is also required for interaction with CDK1, this implies that the function of cyclin $\mathrm{B} 1$ on kinetochores depends on the association between cyclin $\mathrm{B} 1$ and $\mathrm{CDK} 1$, and may require the activity of the cyclin B1-CDK1 kinase complex. One possibility is that cyclin B1-CDK1 is targeted to kinetochores through a relatively stable interaction with substrates localized there. However, it remains possible that a CDK1-independent interaction of the cyclin box region of cyclin B1 with the kinetochore is involved. In addition to the role of the $\mathrm{C}$-terminal portion of the molecule, the $\mathrm{N}$-terminal region appears to also play a role in the interaction of cyclin B1 with unattached kinetochores, although not through binding of the NES motif in cyclin B1 to Crm1. The interaction between the $\mathrm{N}$-terminal domain of cyclin B1 and unattached kinetochores is similar to that with chromosome arms, and may therefore involve an interaction with chromatin rather than with components specific to kinetochores. Since cyclin B1 localizes to multiple locations during prometaphase, namely the spindle, centrosomes, chromosome arms and kinetochores, this poses the question of whether there is a common pool of cyclin B1 molecules that can interact with any site or whether individual cyclin B1 molecules are targeted to a specific location, for instance by a distinctive post-translational label.

The components of kinetochores that are required for the interaction of cyclin B1 are as yet unclear. Depletion of Incenp, Aurora B or Plk1 by siRNA did not substantially affect the kinetochore localization of cyclin B1 (data not shown), indicating that they are not required for either the attachment of cyclin B1 to the kinetochore or its regulation. It is likely that the localization of cyclin B1 to kinetochores is controlled jointly with Mad2 and BubR1 through the activity of the dynein/dynactin complex. The mechanism by which the transport of cyclin B1 away from kinetochores is initiated after microtubule attachment is likely to be of significance in understanding how the functions of cyclin B1-CDK1 at kinetochores and in the spindle checkpoint are controlled.

The cyclin B1-CDK1 complex has previously been implicated in NEBD, spindle assembly and chromosome condensation during prometaphase. Here, we found that cyclin B1 is also localized on kinetochores, suggesting a specific function for cyclin B1-CDK1 at these structures. Using vector-based siRNA, we depleted endogenous cyclin B1 and this caused defects in chromosome alignment during mitosis. However, cyclin B1 depletion did not affect the localization of other kinetochore proteins examined so far, suggesting that it is not required for overall kinetochore structure (Supplementary information, figure S9). Moreover, the overall structure of the mitotic spindle appeared to be normal in cyclin B1-depleted cells, although $\mathrm{k}$-fibres were weakened and the inter-kinetochore distance was reduced, implying specific defects in the attachment of microtubules to kinetochores. Remarkably, HeLa cells were able to undergo NEBD, chromatin condensation and gross spindle formation with little or no cyclin B1. This is consistent with a previous report that neither cyclin $\mathrm{B} 1$ nor B2 is essential for NEBD in HeLa cells [41]. That cyclin B1 knockdown does, however, cause clear defects in chromosome attachment and alignment indicates the functions of cyclin B1-CDK1 in regulation of this process. One caveat to our RNA interference experiments is that we necessarily knocked down the expression of the entire pool of cyclin B1 and not just those molecules at unattached kinetochores.

\section{Materials and Methods}

\section{Cyclin B1 constructs and RNAi interference}

Cyclin B1 was obtained from HeLa cells by RT-PCR, and it was sequenced and cloned into a pEGFPC1 vector (Clontech). The truncated mutant (residues 1-90aa, 1-200aa, 33-433aa, 120-433aa and 150-433aa) of cyclin B1 was amplified from the full-length cyclin B1 cDNA gene by PCR and cloned into the pEGFPC1 vector (Clontech). Mutants disrupting the binding between cyclin B1 and cdk1 were generated by site-directed mutagenesis and cloned in-frame into a pEGFPC1 vector (Clontech). The DNA fragment for the expression of shRNA targeting the positions 776-796 of human cyclin B1 (NCBI accession number NM 031966) was generated by annealing the sense oligonucleotide GAT CCC CGA AAT GTA CCC TCC AGA AAT TCA AGA GAT TTC TGG AGG GTA CAT TTC TTT TTA and the anti-sense oligonucleotide AGC TTA AAA AGA AAT GTA CCC TCC AGA AAT CTC TTG AAT TTC TGG AGG GTA CAT TTC GGG and ligated to pSuper plasmid.

\section{ATP inhibitior assay}

For the ATP inhibitor studies, HeLa cells expressing GFP-cyclin B1 were rinsed in saline $\left(140 \mathrm{mM} \mathrm{NaCl}, 5 \mathrm{mM} \mathrm{KCl}, 0.6 \mathrm{mM} \mathrm{MgSO}_{4}\right.$, 
$0.1 \mathrm{mM} \mathrm{CaCl}_{2}, 1 \mathrm{mM} \mathrm{Na}_{2} \mathrm{HPO}_{4}, 1 \mathrm{mM} \mathrm{KH}_{2} \mathrm{PO}_{4}, \mathrm{pH} 7.3$ ) to remove tissue culture medium and placed in either saline $\mathrm{g}$ (saline with 4.5 $\mathrm{g} / \mathrm{d}$-glucose) or saline plus $5 \mathrm{mM}$ Azide and $1 \mathrm{mM}$ Dog for $30 \mathrm{~min}$ at $37{ }^{\circ} \mathrm{C}$. Cells were then processed for immunofluorescence. For microtubule depolymerization assays, $2 \mathrm{iM}$ nocodazole was added to all solutions. All chemicals were purchased from Sigma-Aldrich.

\section{Cell culture and synchronization}

HeLa cells were cultured in DMEM (Gibco) with 10\% foetal bovine serum (HyClone Laboratories Inc.), $100 \mathrm{U} / \mathrm{ml}$ penicillin and $100 \mu \mathrm{g} / \mathrm{ml}$ streptomycin (Invitrogen). For the double thymidine block and release experiment, HeLa cells were arrested for $14 \mathrm{~h}$ with 2.5 $\mathrm{mM}$ thymidine (Sigma) with a $12 \mathrm{~h}$ release interval. Arrested cells were either released into fresh medium to follow cell cycle progression or into the medium containing $200 \mathrm{ng} / \mathrm{ml}$ nocodazole (Sigma) to trap the cells at prometaphase.

\section{Cell transfection}

HeLa cells were transfected using a standard calcium-phosphate transfection protocol. For the localization of cyclin B1 on kinetochores, GFP-cyclin B1 vector was transfected into HeLa cells using calcium-phosphate transfection. For depletion of endogenous cyclin B1, GFP-H2B was co-transfected with pSuper-cyclin B1 at a ratio of 1:20 to indicate the pSuper-cyclin B1 transfected cells and show chromosomal dynamics with depletion of cyclin B1.

\section{Live-cell imaging experiment}

For live-cell imaging of exogenous GFP-cyclin B1, HeLa cells were transfected transiently for $30 \mathrm{~h}$. The culture dish was placed onto a heated sample stage within a heated chamber $\left(37^{\circ} \mathrm{C}\right)$ and $5 \%$ $\mathrm{CO}_{2}$. Live-imaging was performed using the plan APO 63'/1.35 objective on the above-described Zeiss $200 \mathrm{M}$ microscopy and axiovert software was used to collect and process data. Images were captured with $200 \mathrm{~ms}$ exposure times in $3 \mathrm{~min}$ intervals. For live-cell imaging of cells with depletion of cyclin B1, HeLa cells transfected with GFP-H2B were used. Cells were co-transfected with pSuper-cyclin $\mathrm{B} 1$ and GFP-H2B for $48 \mathrm{~h}$ and the cells were observed as above.

\section{Immunofluorescence and chromosome spread}

To obtain the kinetochore localization of endogenous cyclin B1, HeLa cells were extracted firstly by $0.2 \%$ Triton X 100 in PEM buffer (100 mM Pipes, pH 6.8, $10 \mathrm{mM}$ EGTA, $5 \mathrm{mM} \mathrm{MgCl}_{2}$ ), then fixed in $4 \%$ paraformaldehyde (PFA) in the same buffer. In other cases, HeLa cells were fixed in cold methanol for $5 \mathrm{~min}$, and then immunostained with antibodies. To obtain the kinetochore localization of GFP-cyclin B1, HeLa cells expressing GFP-cyclin B1 were fixed in pre-cooled methanol and immunostained with antibodies. For chromosome spreads, HeLa cells were synchronized in G1/S phase by thymidine treatment for $16 \mathrm{~h}$, and then released for $12 \mathrm{~h}$ to enrich mitosis cells. Mitotic cells were collected by shaking, and then dehydrate with 55 $\mathrm{mM} \mathrm{KCl}$ for $15 \mathrm{~min}$ at $37^{\circ} \mathrm{C}$. The cells were centrifuged onto glass slides at $2000 \mathrm{rpm}$, and the slides fixed by methanol or PFA followed by immunostaining with antibodies as indicated. To analyse changes in cyclin B1-depleted cells, HeLa cells with depletion of cyclin B1 were fixed with pre-cooled methanol for $5 \mathrm{~min}$ at room temperature and immunostained routinely with the following primary antibodies: mouse monoclonal antibodies against $\alpha$-tubulin (Sigma), Aurora B (BD bioscience), HEC1 (Abcam), goat antibodies against BUB1 (Santa Cruz), CENP-E (Santa Cruz), rabbit polyantibody against
CENP-A (Upstate), and rabbit antibodies against MAD2 (Abcam), Plk1 (raised by injecting rabbits), followed by relevant fluorescencedye conjugated secondary antibodies. TRITC goat anti-rabbit IgG, FITC goat anti-mouse IgG and TRITC goat anti-mouse IgG were from Jackson Immunoresearch, while FITC rabbit anti-goat IgG was from Santa Cruz.

\section{Cell extracts and Western blotting}

After being resolved on 10\% SDS-PAGE gels, the protein samples were transferred onto nitrocellulose membrane in transfer buffer $(25$ $\mathrm{mM}$ Tris, $192 \mathrm{mM}$ glycine and $20 \%$ methanol) for $1 \mathrm{~h}$ at $100 \mathrm{~V}$. The filters were then blocked in TTBS [20 mM Tris- $\mathrm{HCl}(\mathrm{pH} 7.4), 500$ $\mathrm{mM} \mathrm{NaCl}$ and $0.3 \%$ Tween-20] containing 5\% non-fat milk overnight at $4{ }^{\circ} \mathrm{C}$ and probed with primary antibodies diluted in TTBS containing $5 \%$ non-fat milk, monoclonal antibody against cyclin B1 (Upstate) diluted 1:1000, goat polyantibody against cyclin A2 (Santa Cruz) diluted 1:1000, monoclonal antibody against cyclin B2 (Santa Cruz) diluted 1:1000, rabbit serum against GFP (generated by injecting rabbit) diluted 1:5000. The filters were then washed 3 times with TTBS and blocked again for $30 \mathrm{~min}$ in TTBS containing 5\% non-fat milk at room temperature. The filters were then incubated with horseradish peroxidase (HRP)-conjugated goat anti-rabbit or goat anti-mouse secondary antibodies (Jackson immunoresearch) diluted 1:6000 in TTBS containing 5\% non-fat milk at room temperature. After final washes in TTBS, the filters were developed for visualization by enhanced chemiluminescence (Sigma) and X-ray film.

\section{Acknowledgements}

We gratefully thank all the other members in our laboratories for useful comments. This work was supported by funds from the National Natural Science Foundation of China (NSFC) (30421004, 30225016, 30330200 and 30671063), the National Basic Research Program of China (2004CB720003 and 2006CB0D0100) and the National Key Scientific Program of China (2007CB914502). PRC is the holder of a Royal Society-Wolfson Research Merit Award.

\section{References}

1 Nigg EA. Mitotic kinases as regulators of cell division and its checkpoints. Nat Rev Mol Cell Biol 2001; 2:21-32.

2 Malumbres M, Barbacid M. Mammalian cyclin-dependent kinases. Trends Biochem Sci 2005; 30:630-641.

3 Clute P, Pines J. Temporal and spatial control of cyclin B1 destruction in metaphase. Nat Cell Biol 1999; 1:82-87.

4 Ookata K, Hisanaga S, Okumura E, Kishimoto T. Association of p34cdc2/cyclin B complex with microtubules in starfish oocytes. J Cell Sci 1993; 105(Part 4):873-881.

5 Ookata K, Hisanaga S, Okano T, Tachibana K, Kishimoto T. Relocation and distinct subcellular localization of p34cdc2-cyclin B complex at meiosis reinitiation in starfish oocytes. EMBO J 1992; 11:1763-1772.

6 Bailly E, Pines J, Hunter T, Bornens M. Cytoplasmic accumulation of cyclin B1 in human cells: association with a detergentresistant compartment and with the centrosome. J Cell Sci 1992; 101(Part 3):529-545. 
7 Pines J, Hunter T. Human cyclins A and B1 are differentially located in the cell and undergo cell cycle-dependent nuclear transport. J Cell Biol 1991; 115:1-17.

8 Jackman M, Firth M, Pines J. Human cyclins B1 and B2 are localized to strikingly different structures: B1 to microtubules, B2 primarily to the Golgi apparatus. EMBO J 1995; 14:16461654.

9 Ubersax JA, Woodbury EL, Quang PN, et al. Targets of the cyclin-dependent kinase Cdk1. Nature 2003; 425:859-864.

10 McEwen BF, Hsieh CE, Mattheyses AL, Rieder CL. A new look at kinetochore structure in vertebrate somatic cells using highpressure freezing and freeze substitution. Chromosoma 1998; 107:366-375.

11 Cleveland DW, Mao Y, Sullivan KF. Centromeres and kinetochores: from epigenetics to mitotic checkpoint signaling. Cell 2003; 112:407-421.

12 Maiato H, DeLuca J, Salmon ED, Earnshaw WC. The dynamic kinetochore-microtubule interface. J Cell Sci 2004; 117(Part 23):5461-5477.

13 Chan GK, Liu ST, Yen TJ. Kinetochore structure and function. Trends Cell Biol 2005; 15:589-598.

14 Kline-Smith SL, Sandall S, Desai A. Kinetochore-spindle microtubule interactions during mitosis. Curr Opin Cell Biol 2005; 17:35-46.

15 DeLuca JG, Gall WE, Ciferri C, et al. Kinetochore microtubule dynamics and attachment stability are regulated by Hec1. Cell 2006; 127:969-982.

16 DeLuca JG, Dong Y, Hergert P, et al. Hec1 and nuf2 are core components of the kinetochore outer plate essential for organizing microtubule attachment sites. Mol Biol Cell 2005; 16:519-531.

17 Bomont P, Maddox P, Shah JV, Desai AB, Cleveland DW. Unstable microtubule capture at kinetochores depleted of the centromere-associated protein CENP-F. EMBO J 2005; 24:39273939.

18 Joseph J, Liu ST, Jablonski SA, Yen TJ, Dasso M. The RanGAP1-RanBP2 complex is essential for microtubule-kinetochore interactions in vivo. Curr Biol 2004; 14:611-617.

19 Sillje HH, Nagel S, Korner R, Nigg EA. HURP is a Ran-importin beta-regulated protein that stabilizes kinetochore microtubules in the vicinity of chromosomes. Curr Biol 2006; 16:731-742.

20 Wong J, Fang G. HURP controls spindle dynamics to promote proper interkinetochore tension and efficient kinetochore capture. $J$ Cell Biol 2006; 173:879-891.

21 Musacchio A, Salmon ED. The spindle-assembly checkpoint in space and time. Nat Rev Mol Cell Biol 2007; 8:379-393.

22 Galjart N. CLIPs and CLASPs and cellular dynamics. Nat Rev Mol Cell Biol 2005; 6:487-498.

23 D'Angiolella V, Mari C, Nocera D, Rametti L, Grieco D. The spindle checkpoint requires cyclin-dependent kinase activity. Genes Dev 2003; 17:2520-2525.

24 Goto H, Kiyono T, Tomono Y, et al. Complex formation of Plk1 and INCENP required for metaphase-anaphase transition. Nat Cell Biol 2006; 8:180-187.

25 Qi W, Tang Z, Yu H. Phosphorylation- and polo-box-dependent binding of Plk1 to Bub1 is required for the kinetochore localization of Plk1. Mol Biol Cell 2006; 17:3705-3716.

26 Ahonen LJ, Kallio MJ, Daum JR, et al. Polo-like kinase 1 cre- ates the tension-sensing $3 \mathrm{~F} 3 / 2$ phosphoepitope and modulates the association of spindle-checkpoint proteins at kinetochores. Curr Biol 2005; 15:1078-1089.

27 Matsumura S, Toyoshima F, Nishida E. Polo-like kinase 1 facilitates chromosome alignment during prometaphase through BubR1. J Biol Chem 2007; 282:15217-15227.

28 Elowe S, Hummer S, Uldschmid A, Li X, Nigg EA. Tensionsensitive Plk1 phosphorylation on BubR1 regulates the stability of kinetochore microtubule interactions. Genes Dev 2007; 21:2205-2219.

29 Acquaviva C, Herzog F, Kraft C, Pines J. The anaphase promoting complex/cyclosome is recruited to centromeres by the spindle assembly checkpoint. Nat Cell Biol 2004; 6:892-898.

30 Swaminathan S, Kiendl F, Korner R, et al. RanGAP1*SUMO1 is phosphorylated at the onset of mitosis and remains associated with RanBP2 upon NPC disassembly. J Cell Biol 2004; 164:965971.

31 Rattner JB, Lew J, Wang JH. p34cdc2 kinase is localized to distinct domains within the mitotic apparatus. Cell Motil Cytoskelet 1990; 17:227-235.

32 Howell BJ, McEwen BF, Canman JC, et al. Cytoplasmic dynein/ dynactin drives kinetochore protein transport to the spindle poles and has a role in mitotic spindle checkpoint inactivation. $J$ Cell Biol 2001; 155:1159-1172.

33 Porter ME, Johnson KA. Transient state kinetic analysis of the ATP-induced dissociation of the dynein-microtubule complex. J Biol Chem 1983; 258:6582-6587.

34 Howell BJ, Hoffman DB, Fang G, Murray AW, Salmon ED. Visualization of Mad2 dynamics at kinetochores, along spindle fibers, and at spindle poles in living cells. J Cell Biol 2000; 150:1233-1250.

35 Kobayashi H, Stewart E, Poon R, et al. Identification of the domains in cyclin A required for binding to, and activation of, p34cdc2 and p32cdk2 protein kinase subunits. Mol Biol Cell 1992; 3:1279-1294.

36 Stewart E, Kobayashi H, Harrison D, Hunt T. Destruction of Xenopus cyclins $\mathrm{A}$ and $\mathrm{B} 2$, but not $\mathrm{B} 1$, requires binding to p34cdc2. EMBO J 1994; 13:584-594.

37 Arnaoutov A, Azuma Y, Ribbeck K, et al. Crm1 is a mitotic effector of Ran-GTP in somatic cells. Nat Cell Biol 2005; 7:626632.

38 Bentley AM, Normand G, Hoyt J, King RW. Distinct sequence elements of Cyclin B1 promote localization to chromatin, centrosomes, and kinetochores during mitosis. Mol Biol Cell 2007; 18:4847-4858.

39 Chung E, Chen RH. Phosphorylation of Cdc20 is required for its inhibition by the spindle checkpoint. Nat Cell Biol 2003; 5:748753.

40 Wong OK, Fang G. Cdk1 phosphorylation of BubR1 controls spindle checkpoint arrest and Plk1-mediated formation of the 3F3/2 epitope. J Cell Biol 2007; 179:611-617.

41 Gong D, Pomerening JR, Myers JW, et al. Cyclin A2 regulates nuclear-envelope breakdown and the nuclear accumulation of cyclin B1. Curr Biol 2007; 17:85-91.

(Supplementary Information is linked to the online version of the paper on the Cell Research website.) 